\title{
Esophageal candidiasis in an immunocompetent host: case report and review of literature
}

\author{
Prerna Goyal', Omesh Goyal', Deepinder Chhina ${ }^{3}$, Pavneet Kaur ${ }^{4}$, \\ Aminder Singh ${ }^{4}$, Rajoo Singh Chhina ${ }^{2}$ \\ ${ }^{2}$ Department of Medicine, ${ }^{2}$ Department of G astroenterology, ${ }^{3}$ Department of Microbiology and \\ ${ }^{4}$ Department of Pathology, Dayanand Medical College and Hospital, Ludhiana, Punjab
}

\begin{abstract}
Infection by Candida species is the most common cause of infectious esophagitis in adults. Esophageal candidiasis (EC) occurs most commonly in immunocompromised hosts, such as those with human immunodeficiency virus infection. However, EC may also occur in immunocompetent individuals with or without apparent predisposing risk factors and symptoms. We report a case of EC incidentally discovered in an immunocompetent middle age male who underwent esophagogastro-duodenoscopy for dyspeptic symptoms. The only potential risk factor was long-term use of alcohol. Treatment with fluconazole led to complete resolution of EC.
\end{abstract}

Keywords: Esophageal candidiasis, fluconazole, immunocompetent

\section{INTRODUCTION}

Esophageal candidiasis (EC) is one of the most common opportunistic infections in patients with impaired cellular immunity, such as human immunodeficiency virus (HIV) infection. ${ }^{[1]}$ However, EC has also been reported to occur in immunocompetent individuals with or without apparent predisposing risk factors and symptoms. ${ }^{[2.5]}$ Various risk factors implicated to cause $\mathrm{EC}$ in immunocompetent individuals include broad-spectrum antibiotics, proton-pump inhibitors (PPI), H2-receptor antagonists, esophageal diseases such as noninfectious esophagitis or achalasia, and use of corticosteroid/cytotoxic drugs. ${ }^{[2.5]}$ Other conditions associated with an increased incidence of EC include alcoholism, malnutrition, and advanced age. ${ }^{[2-5]}$ Most of the patients detected to have EC on esophago-gastro-duodenoscopy (EGD) are asymptomatic, and typical esophageal symptoms are uncommon. ${ }^{[2]}$ We report a case of EC incidentally discovered in an immunocompetent middle age male who underwent EGD for dyspeptic symptoms.

Corresponding author: Dr. Omesh Goyal

E-mail: goyalomesh@yahoo.co.in

Received: 19-07-2016

Accepted: 18-10-2016

How to cite this article: Goyal P, Goyal O, Chhina D, Kaur P, Singh A, Chhina RS. Esophageal candidiasis in an immunocompetent host: case report and review of literature. J Gastrointest Infect, 2016; 6:54-56

\section{CASE REPORT}

A 49 year old male patient presented to gastroenterology OPD with complaints of epigastric discomfort since one month. There was no history of nausea, vomiting, hematemesis, melena, diarrhea, constipation, weight loss, jaundice or fever. He used to consume about 100 gram of alcohol daily since last 20 years. There was no history of smoking, or drug abuse. He was taking PPI (Tab esomeprazole $40 \mathrm{mg}$, once daily) since 3 weeks. He denied any use of analgesics or antibiotics in the last one year. Physical examination revealed a well nourished, moderately built person. He had a blood pressure of 124/80 $\mathrm{mm} \mathrm{Hg}$ and heart rate of 74 beats per minute. His oral cavity was normal with no signs of infection or thrush. He had mild epigastric discomfort with no rebound tenderness or guarding. Rest of physical examination was unremarkable. Laboratory investigations revealed $62 \mathrm{IU} / \mathrm{ml}$ alanine transaminase and $85 \mathrm{IU} / \mathrm{ml}$ aspartate transaminase. Other investigations including serum bilirubin, albumin, complete blood counts, renal function tests, thyroid function test and glycosylated hemoglobin were normal. Ultrasonography of abdomen and pelvis revealed hepatomegaly with fatty liver. Further, EGD was performed, which revealed multiple whitish plaques throughout esophagus with underlying ulceration (Fig.1). Stomach and duodenum were normal. Brushings from the lesions revealed spores and pseudohyphae of Candida infiltrating the mature squamous cells at places (Fig. 2). He was further investigated to rule out immunocompromised state. $\mathrm{HBsAg}$, anti-HCV, 


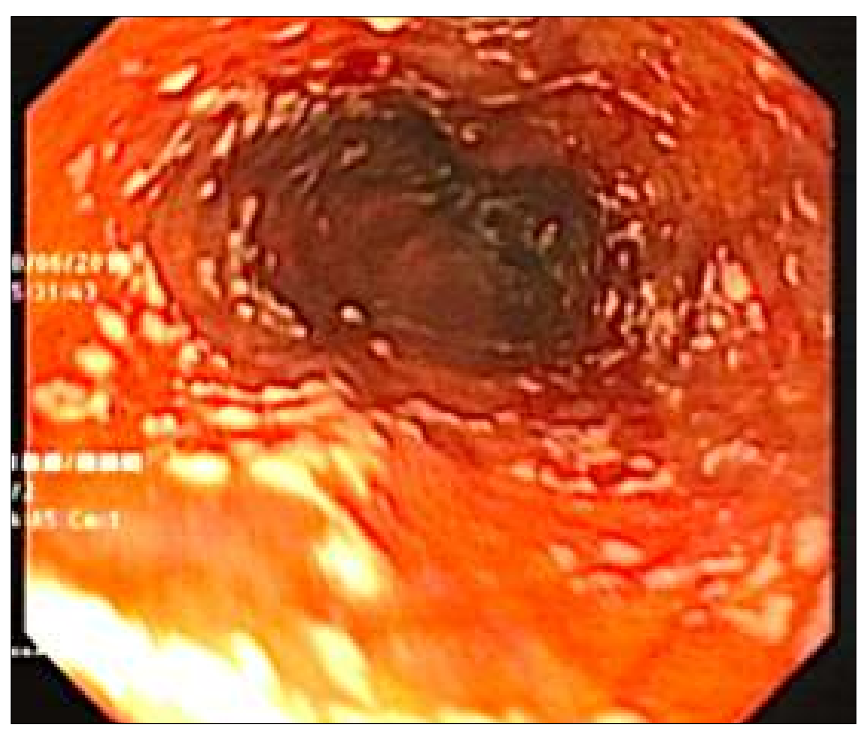

Fig. 1: Endoscopic image showing multiple whitish plaques in esophagus suggestive of esophageal candidiasis

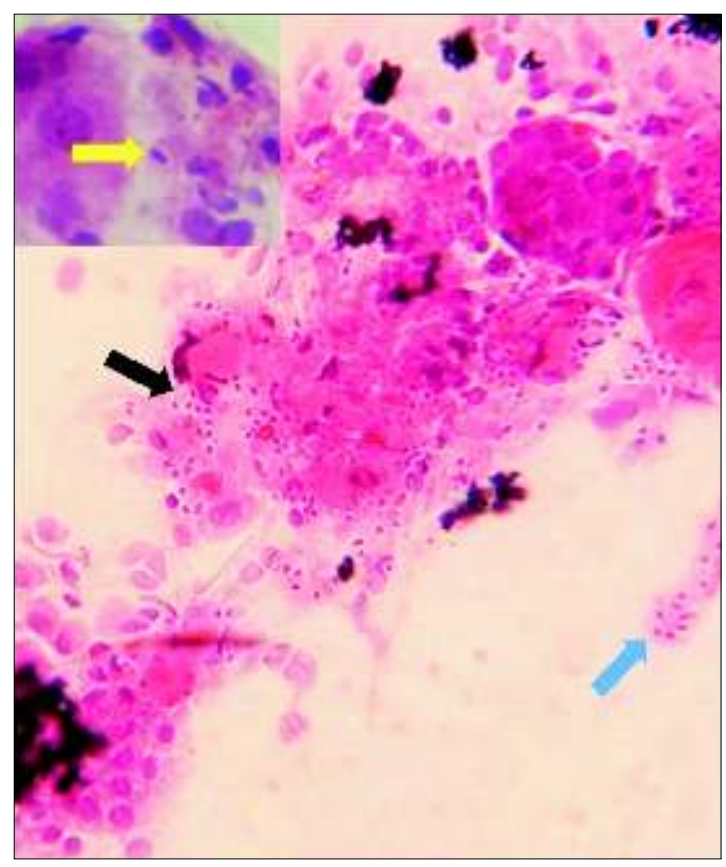

Fig. 2: Spores and pseudohyphae of Candida infiltrating the mature squamous cells at places (Black \& blue arrows; Hematoxylin \& Eosin $x 100)$. Inset showing high power view of Candida (Yellow arrow; Hematoxylin \& Eosin x400)

anti-HIV, syphilis screen, anti-nuclear antibodies and Tlymphocyte counts were normal. He was treated with Tab fluconazole $200 \mathrm{mg}$ once daily for two weeks. Repeat endoscopy performed three weeks later showed complete resolution of esophageal candidiasis.

\section{DISCUSSION}

Esophageal candidiasis has been commonly reported to occur in immunocompromised patients, especially those infected with HIV infection. ${ }^{[1]}$ However, the regular use of screening EGD has led to more frequent diagnosis of EC in healthy individuals. ${ }^{[6]}$ The prevalence of EC among patients undergoing EGD has varied from $0.32 \%(281 / 88125)$ by Choi et $a l^{[2]}$ and $1.17 \%(41 / 3501)$ by Naito et al ${ }^{[7]}$ to $8.7 \%$ by Kakati et $a l^{[8]}$ from India.

The development of EC is a two-step process consisting of colonization of the esophagus and subsequent invasion of the epithelial layer. ${ }^{[9]}$ It is already well accepted that Candida are known to colonize the esophagus of $20 \%$ of healthy adults. Once colonization has been established, impaired cellular immunity, such as due to HIV infection, permits invasion of the epithelial layer. ${ }^{[9]}$

The usual causative agent is Candida albicans, but other Candida species have also been isolated from cases of esophagitis. In a recent report from India, C. albicans was recovered from the majority of patients $(52.1 \%)$, followed by C. tropicalis (24\%), C. parapsilosis (13.4\%), C. glabrata $(6.9 \%)$ and $C$. krusei $(3.6 \%) .^{[8]}$ Alarmingly, among the $C$. albicans isolates $8.6 \%$ were resistant to fluconazole.

In immunocompetent persons, various risk factors for EC have been identified. PPI, H2-receptor antagonists, and prior vagotomy may increase the risk of EC by elevation of gastric $\mathrm{pH}$ and altering the colonization of the esophagus by oral cavity bacteria and yeast. ${ }^{[2,3]}$ Broad-spectrum antibiotics may eliminate certain bacteria that inhibit fungal growth, thereby allowing overgrowth and colonization of the Candida species. ${ }^{[2]}$ Corticosteroids and various cytotoxic drugs predispose to candidiasis by suppressing both lymphocyte and granulocyte function. ${ }^{[2]}$ EC has also been seen in cases of functional or mechanical obstruction of the esophagus, where stasis leads to excessive growth of the fungus. ${ }^{[2,3]}$ Previously, diabetes was considered as risk factor for EC because of impaired immunity and stasis of esophageal contents. ${ }^{[2]}$ However, most of the cases of diabetes-related EC reported have been associated with chronic poor glycemic control (hyperglycemia more than 2 years) and combined with secondary factors, such as those causing an immuno compromised state. ${ }^{[2]}$ Our case had two potential risk factors for EC i.e long standing alcohol intake and use of acid suppressing medications.

As in our case, esophageal symptoms are more frequently absent in individuals with EC. In studies by Choi et al $^{[2]}$ and Naito et $a l,{ }^{[7]}$ about two-thirds of the patients had no symptoms. In those who were symptomatic, variable gastrointestinal symptoms included epigastric discomfort, dyspepsia, nausea etc. Classical symptoms of infectious 
esophagitis, such as dysphagia, odynophagia and chest discomfort have been reported in only about $10 \%$ patients. $^{[2,7]}$ It is questionable whether the gastrointestinal symptoms are associated with EC and whether this has a clinical significance. On follow-up, about three-fourth of the cases of EC have been shown to disappear spontaneously. ${ }^{[2]}$

The endoscopic appearance of Candida esophagitis ranges from a few superficial white plaques on the mucosal surface to a dense, thick pseudomembrane composed of desquamated squamous epithelial cells, fungus, and fibrin. Histologically, in the presence of invasive candidal esophagitis, Candida species are seen along with squamous cells and invading hyphae on smears.

Although most cases of EC may remain silent and invasion of esophageal wall is usually limited to the superficial epithelium, extensive tissue necrosis and ulceration resulting in esophageal perforation is possible. ${ }^{[10]}$ There have been a few reported cases of esophageal perforation associated with Candida infection, and most of these predominantly occurred in immunocompromised hosts, such as transplanted or leukemia patients. It is possible that neutropenia, irradiation and chemotherapy such as methotrexate might have led to mucosal disruption, thereby facilitating deeper invasion of the esophagus by Candida ${ }^{[10]}$ In immunocompetent hosts, chronic alcohol consumption and long-standing gastroesophageal reflux may increase the risk of transmural invasive Candida infection and esophageal perforation. $^{[10]}$

Our case highlights the fact that EC can occur in immunocompetent persons without esophageal symptoms. Confirmation by histopathology and adequate treatment is warranted to prevent complications, especially in persons with underlying risk factors.

CONFLICT OF INTEREST: There is no potential conflict of interest

\section{REFERENCES}

1. Laine L, Bonacini M. Esophageal disease in human immunodeficiency virus infection. Arch Intern Med. 1994;154:1577-82.

2. Choi JH, Lee CG, Lim YJ, Kang HW, Lim CY, Choi JS. Prevalence and risk factors of esophageal candidiasis in healthy individuals: a single center experience in Korea. Yonsei Med J. 2013;54:160-5.

3. Baehr PH, McDonald GB. Esophageal infections: risk factors, presentation, diagnosis and treatment. Gastroenterology. 1994;106:509-32.

4. Ahmed ME, Elfeil MS, Ali IA, Abdelrahim MI, Tamim AA, Khalid MK. Oesophageal candidiasis in an immunocompetent adult, an adverse effect of antibiotic therapy following cardiac surgery, case report and review of literature. East Afr Med J. 2012;89:246-9.

5. Vazquez JA. Mucosal candidiasis. Infect Dis Clin N Am 2002;16:793-820.

6. Cuenca-Estrella M1, Verweij PE, Arendrup MC, Arikan-Akdagli S, Bille J, Donnelly JP et al. ESCMID guideline for the diagnosis and management of $\mathrm{C}$ andida diseases 2012: diagnostic procedures. Clin Microbiol Infect. 2012;18(S7):9-18.

7. Naito Y, Yoshikawa T, Oyamada H, Tainaka K, Morita Y, Kogawa T et al. Esophageal candidiasis. Gastroenterol Jpn. 1988;23:36370.

8. Kakati B, Kotwal A, Biswas D, Sahu S. Fluconazole Resistant Candida Oesophagitis in Immunocompetent Patients: Is Empirical Therapy Justifiable? J Clin Diagn Res. 2015;9:16-8.

9. Hoshika K, lida M, Mine H. Esophageal Candida infection and adherence mechanisms in the non immunocompromised rabbit. J Gastroenterol. 1996;31:307-13.

10. Tran HA, Vincent JM, Slavin MA, Grigg A. Esophageal perforation secondary to angioinvasive Candida glabrata following hemopoietic stem cell transplantation. Clin Microbiol Infect. 2003;9: 1215-8. 